\title{
Premature Ejaculation (lifelong and acquired)
}

Ege Can Serefoglu, Chris G. McMahon, Marcel D. Waldinger, Stanley E. Althof, Alan Shindel, Ganesh Adaikan, Edgardo F. Becher, John Dean, Francois Giuliano, Wayne J.G. Hellstrom, Annamaria Giraldi, Sidney Glina, Luca Incrocci, Emmanuele Jannini, Marita McCabe, Sharon Parish, David Rowland, Robert Taylor Segraves, Ira Sharlip, Luiz Otavio Torres

\section{Source}

Ege Can Serefoglu, Chris G. McMahon, Marcel D. Waldinger, Stanley E. Althof, Alan Shindel, Ganesh Adaikan. (2014). An Evidence-Based Unified Definition of Lifelong and Acquired Premature Ejaculation: Report of the Second International Society for Sexual Medicine Ad Hoc Committee for the Definition of Premature Ejaculation. Sexual

Medicine, vol. 2 (2), 41-59.

A male sexual dysfunction characterized by the following:

1. Ejaculation that always or nearly always occurs prior to or within about 1 minute of vaginal penetration (lifelong PE) or a clinically significant and bothersome reduction in latency time, often to about 3 minutes or less (acquired PE).

2. The inability to delay ejaculation on all or nearly all vag inal penetrations.

3. Negative personal consequences, such as distress, bother, frustration, and/or the avoidance of sexual intimacy. 